\title{
ON NORMS OF COMPOSITION OPERATORS ON WEIGHTED HARDY SPACES
}

Amenah Essa Shamakhi * and Sumitra Dalal

Dept. of Mathematics, Jazan University, K.S. A

Email \# ah-eh-sh@hotmail.com

\section{ABSTRACT:}

The computation of composition operator on Hardy spaces is very hard. In this paper we propose a norm of a bounded composition operator on weighted Hardy spaces $H^{2}(\beta)$ induced by a disc automorphism by embedding the classical Hardy space. The estimate obtained is accurate in the sense that it provides the exact norm for particular instances of the sequence $\beta$. As an application of our results, an estimate for the norm of any bounded composition operator on $H^{2}(\beta)$ is obtained.

\section{INTRODUCTION:}

Let $D$ denote the open unit disc of the complex plane. For each sequence of positive numbers

$\beta=\left\langle\beta_{n}\right\rangle$, the weighted Hardy space $H^{2}(\beta)$ consists of analytic functions $f(z)=\sum_{n=0}^{\infty} a_{n} z^{n}$ on $D$ for which the norm

$\|f\|_{\beta}=\left(\sum_{n=0}^{\infty}\left|a_{n}\right|^{2} \beta_{n}^{2}\right)^{1 / 2}$ is finite. Since the function $f(z)=\sum_{n=0}^{\infty}\left(n \beta_{n}\right)^{-1} z^{n}$ is in $H^{2}(\beta)$, we have $\limsup \beta_{n}^{-1 / n} \leq 1$, where the f norm is proposed from the following inner product

$\left\langle\sum_{n=0}^{\infty} a_{n} z^{n}, \sum_{n=0}^{\infty} b_{n} z^{n},\right\rangle=\sum_{n=0}^{\infty} a_{n} \overline{b_{n}} \beta_{n}^{2}$, and the monomial $\left\{z^{n}\right\}$ form a complete orthogonal system. Consequently, the polynomials are dense in $H^{2}(\beta)$.

Observe that particular instances of the sequence $\beta=\left\langle\beta_{n}\right\rangle$ yield well known Hilbert spaces of analytic functions. Indeed, $\beta_{n}=1$ corresponds to the Hardy space $H^{2}(D)$. If $\beta_{n}=(n+1)^{1 / 2}$ the norm obtained is equivalent to the one in the Dirichlet space $D$ or if $\beta_{n}=(n+1)^{-1 / 2}$, we get the Bergman space $A^{2}$. If $\beta=(n+1)^{q}$ for a real number $q$, the spaces $H^{2}(\beta)$ are known as weighted Dirichlet spaces or $S_{v}$ spaces (see $[8,10,11,19]$, few to mention).

If $\varphi$ is an analytic function on $D$ with $\varphi(D) \subset D$, the equation $C_{\varphi} f=f \circ \varphi$ defines a composition operator $C_{\varphi}$ on $H(D)$, the space of all holomorphic functions on the unit disc. Indeed, $C_{\varphi}$ is a bounded operator on $H(D)$ endowed with the topology of uniform convergence on compacta. However, it is an open problem to determine those holomorphic self maps of $D$ inducing bounded composition operators on $H^{2}(\beta)$. For instance, many composition operators are unbounded when the sequence of weights decreases too fast, that is, the spaces are "too large" [15 or 8 thm 5.2] In this work, we are interested in finding the norm of bounded composition operators on $H^{2}(\beta)$. Our unique requirement on the space $H^{2}(\beta)$ will be that the Hardy space $H^{2}(D)$ is continuously embedded in it. Note that for $v \geq 0$, the weighted Dirichlet space $S_{v}$ satisfies our requirement. A first attempt to estimate the norm of a bounded $C_{\varphi}$ on $H^{2}(\beta)$ is to assume that $\varphi$ is an automorphism of the unit disc $\mathrm{D}$. In such a case, we estimate it by considering a particular semigroup of operators. At this respect, let us remark that in most cases where the norm of $H^{2}(\beta)$ ) can be expressed in terms of an integral, the norm of bounded composition operators induced by disc automorphisms may be 
estimated by a standard procedure involving a change of variables [8] ( Needless to say that such a procedure does not apply in our case because our requirement on $H^{2}(\beta)$ is rather general $\left\langle\beta_{n}\right\rangle$ is not assumed to be a moment sequence). Finally, we just point out that computing the exact norm of a composition operator, even in the classical Hardy space $H^{2}(D)$, is an open problem which seems to be far from being solved. In this respect, we refer to [2,5,12,13,14] for related questions concerning the norm of composition operators.

\section{Norm of composition operators on $H^{2}(\beta)$}

In this section, we estimate the norm of a bounded composition operator induced by a disc automorphism on any weighted Hardy space $H^{2}(\beta)$ which contains $H^{2}(D)$ as well as we estimate the norm of a any bounded composition operator in $H^{2}(\beta)$, First, we begin by recalling that any automorphism $\varphi$ of the unit disc $D$ is expressed by the formula

$\varphi(z)=e^{i \theta} \frac{w-z}{1-\bar{w} z}$, where $\theta \in(-\pi, \pi), w \in D$ In addition, automorphisms of $D$ can be classified according to their fixed points. Indeed, if $\varphi$ is not the identity function, then by solving a two degree equation the following can be proved [17].

i) $\varphi$ has an unique fixed point $z$ if and only if $|w|=\cos (\theta / 2)$. In such a case, $z$ belongs to the boundary of the unit disc $\partial D \partial \mathrm{D}$ and $\varphi$ is called parabolic.

ii) $\varphi$ has two fixed points $y$ and $z$ with $|z|<1$ if and only if $|w|<\cos (\theta / 2)$. In such a case, $q$ belongs to the complement of the closure of $D$ in the extended plane $C_{\infty}=C \cup\{\infty\}$ and $\varphi$ is called elliptic.

iii) $\varphi$ has two fixed points $y$ and $z$ with $|z|=1$ if and only if $|w|>\cos (\theta / 2)$. In such a case, it also holds that $|q|=1$ and $\varphi$ is called hyperbolic. Note that if $\varphi$ is a non-identity disc automorphism, then there exists a unique $r \in(0,1)$ so that $\varphi$ may be expressed as

$\psi_{\mu 1} \circ \alpha_{r} \circ \psi_{\mu 2}$, where $\alpha_{r}$ is the hyperbolic automorphism

$$
\alpha_{r}(z)=\frac{z+r}{1+r z}
$$

and $\psi_{\mu_{i}}=\mu_{i} z, i=1,2$ are disc rotations (i.e. $\left|\mu_{i}\right|=1$ ). In fact, observe that $r=|\varphi(0)|$. In addition, note that a simple computation shows that any disc rotation $\psi_{\mu}(z)=\mu z$ with $|\mu|=1$ induces a bounded composition operator in each weighted Hardy space $H^{2}(\beta)$. Moreover, it holds that $\left\|C_{\psi_{\mu}}\right\|_{H^{2}(\beta)}=1$ and $C_{\psi_{\mu}}$ is an invertible operator on $H^{2}(\beta)$ with $C_{\psi_{\mu}}^{-1}=C_{\psi_{1} / \mu}$. Hence, we may deduce the following.

Proposition 2.1. Let $H^{2}(\beta)$ be a weighted Hardy space and $\varphi$ a disc automorphism. Then $C_{\varphi}$ is bounded on $H^{2}(\beta)$ if and only if the composition operator induced by the hyperbolic automorphism $\alpha_{|\varphi(0)|}(z)=(z+|\varphi(0)|) /(1+|\varphi(0)|) \quad$ is bounded $\quad$ on $\quad H^{2}(\beta)$. Moreover, in such a case $\left\|C_{\varphi}\right\|_{H^{2}(\beta)}=\left\|C_{\alpha \mid \varphi(0)}\right\|_{H^{2}(\beta)}$. 
Note that there is no extra assumption on the weighted Hardy space $H^{2}(\beta)$ ) in Proposition 2.1. In addition, observe as a consequence that we only need to compute the norm in $H^{2}(\beta)$ of those bounded composition operators induced by the hyperbolic automorphisms $\alpha_{r}$ with $0<r<1$.

\section{A lower bound.}

In this subsection, we will show the following:

Proposition 3.1. Let $H^{2}(\beta)$ be a weighted Hardy space which contains $H^{2}(D)$. Let $0<r<1$ and $\alpha_{r}(z)=\frac{z+r}{1+r z}$ a hyperbolic disc automorphism so that $C_{\alpha_{r}}$ is bounded on $H^{2}(\beta)$. Denote by $a=\inf \left\{s \in[0, \infty):(1-z)^{-s} \notin H^{2}(\beta)\right\}$. Then $\left\|C_{\alpha_{r}}\right\|_{H^{2}(\beta)} \geq\left(\frac{1+r}{1-r}\right)^{a}$.

In order to prove Proposition 2.2, we will exhibit eigenfunctions of $C_{\alpha_{r}}$ in $H^{2}(\beta)$. In what follows, log will denote the principal branch of the natural logarithm function.

Lemma 3.2 Let $H^{2}(\beta)$ be a weighted Hardy space which contains $H^{2}(D)$. Let $0<r<1$ and $\alpha_{r}=\frac{z+r}{1+r z}$ a hyperbolic disc automorphism so that $C_{\alpha_{r}}$ is bounded on $H^{2}(\beta)$. Denote by $a=\inf \left\{s \in[0, \infty):(1-z)^{-s} \notin H^{2}(\beta)\right\}$. If $-a<\operatorname{Re} w<a$, then $K_{w}(z)=\left(\frac{1+z}{1-z}\right)^{w}=\exp \left(w \log \frac{1+z}{1-z}\right)$

is an eigenfunction in $H^{2}(\beta)$ for $C_{\alpha_{r}}$ corresponding to the eigenvalue $\left(\frac{1+r}{1-r}\right)^{w}$.

The proof consists of a computation which follows the lines of that of Lemma 7.3 in [8], with a little bit more care. We include it for the sake of completeness.

Proof. First, let us check that $K_{w}$ belongs to $H^{2}(\beta)$ for any $-a<\operatorname{Re} w<a$,. Note that $K_{w}$ is a branch of the function $\left(\frac{1+z}{1-z}\right)^{\operatorname{Re} w}\left(\frac{1+z}{1-z}\right)^{i \operatorname{Im} w}$

and that the bounded analytic function $\left(\frac{1+z}{1-z}\right)^{i \operatorname{Im} w}$ belongs to $H^{2}(\beta)$. Therefore, by a result of Shields (see [18, Theorem 10]), it is a multiplier of $H^{2}(\beta)$. Hence, it is enough to determine when $\left(\frac{1+z}{1-z}\right)^{\operatorname{Re} w}$ belongs to $H^{2}(\beta)$. On the other hand, observe that the same argument applies to $(1-z)^{\operatorname{Re} w}$ as far as $\operatorname{Re} w>0$. Hence, it suffices to determine for which complex numbers $w$ with $\operatorname{Re} w>0$ the functions $\left(\frac{1}{1-z}\right)^{\operatorname{Re} w}$ and $\left(\frac{1}{1+z}\right)^{\operatorname{Re} w}$ belong to $H^{2}(\beta)$. Fix $w \in C$ with $0<\operatorname{Re} w<a$ and consider the Taylor series of $\left(\frac{1}{1-z}\right)^{\operatorname{Re} w}$, i.e., 
$\left(\frac{1}{1-z}\right)^{\operatorname{Re} w}=\sum_{n=0}^{\infty} \frac{\Gamma(n+\operatorname{Re} w)}{\Gamma(n+1) \Gamma(\operatorname{Re} w)} z^{n},|z|<1$

where $\Gamma$ denotes the Euler Gamma function. Obviously, $\left(\frac{1}{1-z}\right)^{\operatorname{Re} w} \in H^{2}(\beta)$ if and only if the series $\sum_{n=0}^{\infty} \frac{\Gamma(n+\operatorname{Re} w)}{\Gamma(n+1) \Gamma(\operatorname{Re} w)} \beta_{n}^{2}$ is convergent. Choosing $\operatorname{Re} w<s<a$, it follows that $(1-z)^{-s} \in H^{2}(\beta) \quad$ and therefore, we may deduce that the series in the last display converges. Analogously, it follows that $\left(\frac{1}{1+z}\right)^{\text {Rew }} \in H^{2}(\beta)$, which yields the statement of the lemma.

With Lemma 2.3 at hand, the proof of Proposition 2.2 follows, since the norm of a bounded operator is greater than its spectral radius.

\section{Upper bound.}

In this subsection, we will find an upper bound for the norm of a bounded composition operator induced by an automorphism in $H^{2}(\beta)$. Our approach will rely on semi-group techniques and a detailed analysis of the numerical range of its infinitesimal generator. First, let us fix $0<r<1$ and $\alpha_{r}=\frac{z+r}{1+r z}$ a hyperbolic disc automorphism so that $C_{\alpha_{r}}$ is bounded on $H^{2}(\beta)$. Because of Proposition 2.1, to estimate the norm of a bounded composition operator induced by an automorphism in $H^{2}(\beta)$ it suffices to estimate the norm of $C_{\alpha_{r}}$ in $H^{2}(\beta)$. Observe that the change of variable $t=\frac{1}{2} \log \frac{1+r}{1-r}$, that is, $r=\tanh t$, gives us that $C_{\alpha_{r}}$ is a semigroup of of operators, i.e., $T_{t}=C_{\alpha \tanh t}$,

since $\alpha_{r} \alpha_{s}=\alpha_{(r+s) /(1+r s)}$. It holds that $\left\{T_{t}\right\}$ is a strongly continuous semigroup (see [4] ). Its infinitesimal generator A can be calculated by (writing $e_{n}(z)=z^{n}$ ) as

$$
\begin{aligned}
\left(A e_{n}\right)(z) & =\lim _{t \rightarrow 0} t^{-1}\left(T_{t}-1\right) e_{n}(z) \\
& =\lim _{t \rightarrow 0} t^{-1}\left(\left(\frac{z+\tanh t}{1+(\tanh t) z}\right)-z^{n}\right) \\
& =n z^{n-1}-n z^{n+1},
\end{aligned}
$$

Thus, $(A f)(z)=f^{\prime}(z)\left(1-z^{2}\right)$. Note that $A$ is a closed (unbounded) operator. Some spectral properties of $C_{\alpha_{r}}$ can be deduced from the properties of $A$, but what is more useful here is the fact that the numerical range $W(A)$ of $A$ determines the growth rate of the semigroup. This is defined by $W(A)=\{(A f, f): f \in D(A),\|f\|=1\}$, where $D(A)$ is the domain of $A$, and it is known (see for example the book of Davies [9, Sec. 8.3]) that if $A$ is the generator of a $C_{0}$ semigroup $\left(T_{t}\right)_{t \geq 0}$, then $\left\|T_{t}\right\| \leq 1$ for all $t \geq 0$ if and only if

$$
W(A) \subseteq\{z \in C: \operatorname{Re} z \leq 0\}
$$

By replacing $A$ by $A-\gamma I$ I for $\gamma \in R$, we see that 
$\left\|T_{t}\right\| \leq e^{\gamma t}$ for all $t \geq 0$ if and only if $\operatorname{Re} z \leq \gamma$ for all $z \in W(A)$. We proceed to calculate an element of $W(A)$

Let $f(z)=\sum_{n=0}^{\infty} a_{n} z^{n}$ and consider

$(A f, f)_{H^{2}(\beta)}=\left\langle\sum_{N=0}^{\infty} n a_{n} z^{n-1}\left(1-z^{2}\right), \sum_{N=0}^{\infty} a_{m} z^{m}\right\rangle_{H^{2}(\beta)}$.

A little computation shows that $\sup \operatorname{Re} W(A)$ can be written in the following terms, given that $A$ has real coefficients:

$$
\begin{aligned}
A & =\sup \operatorname{Re} W(A) \\
& =\sup \operatorname{Re}\left\{\sum_{n=0}^{\infty}(n+1) \beta_{n}^{2} \overline{a_{n}} a_{n+1}-n \beta_{n+1}^{2} a_{n} \overline{a_{n+1}}: \sum_{n=0}^{\infty} \beta_{n}^{2}\left|a_{n}\right|^{2}=1\right\} .
\end{aligned}
$$

As consequence, we have the following

Proposition 4.1. Let $H^{2}(\beta)$ be a weighted Hardy space which contains $H^{2}(D)$. Let $0<r<1$ and $\alpha_{r}=\frac{z+r}{1+r z}$ a hyperbolic disc automorphism so that $C_{\alpha_{r}}$ is bounded on $H^{2}(\beta)$. If $A$ is given by $(1)$, then

$$
\left\|C_{\alpha_{r}}\right\|_{H^{2}(\beta)} \leq\left(\frac{1+r}{1-r}\right)^{A / 2}
$$

We also get the following result for boundedness of composition operators on automorphism invariant weighted Hardy spaces. Just recall that $H^{2}(\beta)$ is said to be automorphism invariant if $C_{\varphi} \mathrm{C} \phi$ is bounded on $H^{2}(\beta)$ for any disc automorphism $\varphi$.

Corollary 4.2. Let $\beta=\left\{\beta_{n}\right\}$ be a monotone decreasing weight sequence and assume that $H^{2}(\beta)$ is automorphism invariant. Let $\varphi$. be a holomorphic self-map of $D$. Then $C_{\varphi}$ is bounded on $H^{2}(\beta)$ and

$\left\|C_{\alpha_{r}}\right\|_{H^{2}(\beta)} \leq\left(\frac{1+|\varphi(0)|}{1-|\varphi(0)|}\right)^{A / 2}$, where $A$ is given by equation $(1)$.

Proof. The key point is a result proved by Cowen in [7] which states that if $\left\{\beta_{n}\right\}$ is monotone decreasing and $\psi \psi$ is a holomorphic self-map of $D$ with $\psi(0)=0$, then $C_{\psi}$ is bounded on $H^{2}(\beta)$ and $\left\|C_{\psi}\right\|_{H^{2}(\beta)}=1$.

This along with the assumption that $H^{2}(\beta)$ is automorphism invariant and Proposition 2.4 (note that $H^{2}(D) \subset H^{2}(\beta)$ since $\beta$ is monotone decreasing) yields the statement.

Finally, let us point out that while a wide range of moderately sized spaces, including any weighted Dirichlet space, are automorphism invariant, automorphism invariance fails for certain weighted Hardy spaces $H^{2}(\beta)$ that are either too large or too small [15-16].

\section{An application: Computing the norm in some weighted Hardy spaces.}

Since $\operatorname{Re} \overline{a_{n}} a_{n+1}=\operatorname{Re} a_{n} \overline{a_{n+1}}$, the calculation of expression (1) becomes much easier if we have

$(n+1) \beta_{n}^{2}-n \beta_{n+1}^{2} \geq 0, \forall n$. 
as then the supremum can be achieved with real scalars (an). Let us consider $\beta_{n}=1 /(n+1)^{v}$ for $v \geq 0$. As an application of Propositions 2.1 and 2.2, it is possible to compute exactly the norm of composition operators induced by automorphisms of the unit disc in $H^{2}(\beta)$ for a wide range of $v$. As it has been already noted, to compute the norm of composition operators induced by disc automorphisms, we are reduced to computing the norm of those just induced by hyperbolic automorphisms. Considering the inequality

$$
x y \leq \frac{c^{2} x^{2}+d^{2} y^{2}}{2 c d},
$$

For all $x, y \in R$ and $c, d>0$, we obtain that $A$ in $(1)$ is bounded by

$$
A=\sup \operatorname{Re} W(A) \leq \sup \left\{\sum_{n=0}^{\infty} \frac{(n+1) \beta_{n}^{2}-n \beta_{n+1}^{2}}{2 \beta_{n} \beta_{n+1}}\left(\beta_{n}^{2} a_{n}^{2}+\beta_{n+1}^{2} a_{n+1}^{2}\right): \sum_{n=0}^{\infty} \beta_{n}^{2}\left|a_{n}\right|^{2}=1\right\} .
$$

Thus one upper bound is given by

$$
\operatorname{Re} W(A) \leq \sup _{n \geq 0} \frac{(n+1) \beta_{n}^{2}-n \beta_{n+1}^{2}}{2 \beta_{n} \beta_{n+1}}=M_{1} \text { say. }
$$

In our case $\beta_{n}=\frac{1}{(n+1)^{p}}$, so one easily gets

$0 \leq(n+1) \frac{(n+2)^{p}}{(n+1)^{p}}-n \frac{(n+1)^{p}}{(n+2)^{p}} \rightarrow 1+2 v$

as $n \rightarrow \infty$. For $0 \leq v \leq 2$ numerical calculation indicates that the sequence is increasing with $\mathrm{n}$, and so $M_{1}=1+2 v$ . Thus, the upper bound and lower bound coincide, giving the following

$\left\|C_{\alpha_{r}}\right\|=\left(\frac{1+r}{1-r}\right)^{M_{1} / 2}=\left(\frac{1+r}{1-r}\right)^{v+1 / 2}$ for $0 \leq v \leq 2$.

We would like to remark here that the expression (5) does not hold for larger $v$, and in such cases, the best estimates for the lower and upper bounds for the norm are given by Propositions 3.1 and 4.1 , respectively. Indeed, taking and

$$
\begin{aligned}
& f(z)=\frac{(1+32 z)}{\sqrt{2}}, \text { we have } \\
& \|f\|_{H^{2}(\beta)}^{2}=\frac{1}{2}+\frac{1}{2}\left(32^{2}\right) / 2_{10}^{10}=1 \\
& (A f, f)=\left(\left(1-z^{2}\right) 32 / \sqrt{2},(1+32 z) / \sqrt{2}\right)_{H^{2}(\beta)}=16
\end{aligned}
$$

That is $\lambda<16$ there is a $t>0$ such that the inequality

$\left\|T_{t}\right\| \leq e^{\lambda t}$, fails to hold. We can conclude that there is an $r$ for which the inequality

$$
\left\|C_{\alpha_{r}}\right\| \leq\left(\frac{1+r}{1-r}\right)^{v+1 / 2} .
$$

fails to hold with $v=5$, the best exponent is at least 8 in this case. 


\section{References}

[1] . Ahlfors L. V, 1979, Complex Analysis, McGraw-Hill, 1979 (third edition).

[2] Axler S., Jewell N. and Shields A., 1980, The essential norm of an operator and its adjoint, Trans. Amer. Math. Soc. 261 (1980), 159-167.

[3] Appel M. J., Bourdon P. S. and Thrall J. J., 1996, Norms of composition operators in the Hardy space, Experiment. Math. 5 (1996), 111-117.

[4] Berkson E. and Porta H.,1978, Semigroups of analytic functions and composition operators, Michigan Math. J., 25 (1978), no. 1, 101-115.

[5] Bourdon P. S., Fry E. E., Hammond C. and Spoff ord C. H., 2004, Norms of linear-fractional composition operators, Trans. Amer. Math. Soc. 356 (2004), 2459-2480.

[6] Cowen C. C.,1983, Composition operators on H2, J. Operator Theory, 9 (1983), 77-106

[7] Cowen C. C.,1990, An application of Hadamard multiplication to operators on weighted Hardy spaces, Linear Alg. Appl. 133 (1990), 21-32.

[8] Cowen C. C. and MacCluer B. D.,1995, Composition Operators on Spaces of Analytic Functions, CRC Press, 1995.

[9] Davies E. B.,2007, Linear operators and their spectra, Cambridge University Press, 2007.

[10] Galindo P and . Lindstrom M,2010, Essential norm of operators on weighted Bergman spaces of infinite order, J. Oper. Theor. 64 (2010), 387-399.

[11] Gallardo-Gutierrez E. A. and Montes-Rodriguez A.,2004 The role of the spectrum in the cyclic behavior of composition operators, Mem. Amer. Math. Soc. 167 (2004), no. 791, x+81 pp.

[12] Hammond C.,2003, On the norm of a composition operator with linear fractional symbol, Acta Sci. Math. (Szeged) 69 (2003), no. 3-4, 813-82

[13] Hammond C.,2006, Zeros of hypergeometric functions and the norm of a composition operator Comput. Methods Funct. Theory 6 (2006), no. 1, 37-50.

[14] Hammond C. and Patton L. J.,2010, Norm inequalities for composition operators on Hardy and weighted Bergman spaces, Topics in operator theory. Volume 1. Operators, matrices and analytic functions, 265-272, Oper. Theory Adv. Appl., 202, Birkhauser Verlag, Basel, 2010.

[15] Kriete T. L. and . MacCluer B. D,1992, Composition operators on large weighted Bergman spaces, Indiana Univ. Math. J. 41 (1992), no. 3, 755-788.

[16] MacCluer B. D., Zeng X. and Zorboska N.,1996, Composition operators on small weighted Hardy spaces, Illinois J. Math. 40 (1996), no. 4, 662-677.

[17] . Shapiro J. H,1993, Composition operators and classical function theory, Springer-Verlag, 1993.

[18] Shields A. L.,1974, Weighted shift operators and analytic function theory, Topics in Operator Theory, Math. Surveys Monographs, Amer. Math. Soc., Providence, RI 13 (1974), 49-128.

[19] Zorboska N.,1990, Composition operators on Sa spaces, Indiana Univ. Math. J. 39 (1990), 847-857. 